Check for updates

New Delhi, India

Cite this as: $B M J 2021 ; 372:$ n52 http://dx.doi.org/10.1136/bmj.n52 Published: 07 January 2021

\title{
Covid-19: Indian health officials defend approval of vaccine
}

Health officials have defended India's decision to approve a homegrown covid-19 vaccine without efficacy data-a move that triggered criticism amid speculation that regulators may have buckled under a government wish for an Indian vaccine alongside those now authorised in other countries..

Researchers have criticised the decision by the Subject Expert Committee (SEC) under the Central Drugs Standard Control Organisation (CDSCO), the regulatory agency, to grant accelerated approvals to the Oxford AstraZeneca vaccine manufactured in India by the Serum Institute of India and the vaccine from India's Bharat Biotech.

The health ministry has said the campaign to vaccinate around 300 million people from four priority groups-healthcare workers, public workers, people over 50, and those below 50 with comorbidities-would begin next week.

Doctors expect the Oxford AstraZeneca vaccine to be the first to be rolled out. The Serum Institute has said it has stockpiled 50 million doses. Under approval conditions, the Bharat Biotech vaccine would be launched in a "clinical trial mode" with recipients considered equivalent to participants in a single arm trial.

For the SEC review Serum had submitted safety, immunogenicity, and efficacy data on the Oxford AstraZeneca recombinant chimpanzee adenovirus vector vaccine from 23745 participants in clinical trials outside India and safety and immunogenicity data from 1600 participants in India.

The safety and immunogenicity data from the Indian trial was "found comparable with the data from the overseas clinical trials," Venugopal Somani, the drugs controller general of India and chief of the CDSCO said, announcing both approvals on 3 January.

Bharat Biotech, which has developed a whole virion inactivated coronavirus vaccine, submitted safety and immunogenicity data on nearly 800 participants showing that the vaccine is safe and generates a robust immune response, Somani said.

Somani and other health officials have suggested that the approval of the inactivated vaccine would help India with "more options" to combat emerging mutant strains. Over the past two weeks, Indian laboratories have detected over 70 patients in different parts of the country with the fast spreading variant with the $\mathrm{N}_{501} \mathrm{Y}$ mutation.

But researchers say there is no evidence to back the claim that the inactivated vaccine would work better against specific variants. Satyajit Rath, a medical immunologist and a visiting faculty member at the Indian Institute of Science Education and Research in Pune, told The BMJ, "To say a specific vaccine might help against the UK variant is wishful thinking."
Bharat Biotech officials have indicated that the interim results of their efficacy trial involving 25800 participants could be expected after March and health officials have asserted that provisions in Indian drug rules permit accelerated approval under public health emergencies.

Balram Bhargava, director general of the Indian Council of Medical Research which has assisted Bharat Biotech in the development of the vaccine, said, "In a pandemic situation, restricted use is considered on the basis of safety and immunogenicity while efficacy trials are ongoing. Immunogenicity data serves as surrogate for efficacy."

Some researchers have questioned the timing, pointing out that India's active cases of covid-19 have fallen from a peak of a million cases in mid-September to under 230 ooo this week, although on average around 18 ooo new daily cases emerged over the past week.

Gagandeep Kang, professor microbiology at the Wellcome Trust Research Laboratory at the Christian Medical College, Vellore, told The BMJ, "What is not clear to me is why a matter of a few weeks became so important."

The All India People's Science Network, representing multiple scientists' organisations, has described the approval of the Indian vaccine as hasty. In a statement, the network said, "This achievement will be hailed as a major Indian scientific achievement once efficacy data are released-but by this hasty approval, the government has shot itself in the foot."

The network said that the approval raises concerns about "possible political pressure." Researchers have pointed out that the Indian Council of Medical Research had, in July, urged principal investigators to fast track the clinical trials on the vaccine.

India's health minister Harsh Vardhan tweeted on 3 January, “A watershed moment in India's famed battle against covid-19 under the charismatic leadership of Prime Minister Narendra Modi.” 